\title{
Automated STEM/EDS Metrology Characterization of 3D NAND Devices
}

\author{
Zhenxin Zhong ${ }^{1}$, Justin Roller ${ }^{1}$, Oleksii Bidiuk ${ }^{1}$, Jeff Blackwood ${ }^{1}$, Martin Verheijen ${ }^{1}$, Ozan Ugurlu ${ }^{1}$, and \\ Jason Donald ${ }^{1}$ \\ 1. Materials \& Structural Analysis (formerly FEI), Thermo Fisher Scientific, 5350 NE Dawson Creek \\ Drive, Hillsboro, OR 97124
}

The semiconductor and memory industries have experienced a major transition from conventional planar devices to complex 3D architectures, such as 3D NAND and FinFETs. New materials and designs have raised metrology complexity and greatly increased the number of critical dimension measurements required for process control. 3D NAND presents many metrology challenges including low contrast features, high aspect ratio trenches, and holes in multiple stacks. Many technologies, such as STEM/TEM imaging, critical dimension small angle X-ray spectroscopy (CD-SAXS) and through focus scanning optical microscopy, have been explored to address these metrology challenges. The techniques in their present forms haven't adequately achieved the sensitivity and resolution to measure the required critical dimensions of these 3D NAND structures.

Advances in EDS detector technology provide new possibilities in the field of analytical characterization at the nanometer scale and have triggered dramatic progress in the analysis and quantification of light elements with EDS. [1] In this paper, we propose a new technique to characterize CD metrology by using a fully automated STEM/EDS mapping and linescan approach. The acquisition, quantification, and metrology are completely automated which enable the generation of a large volume of repeatable, precise, and consistent data. High volume, automated S/TEM image acquisition and metrology analysis of semiconductor devices have been developed and demonstrated using an FEI Metrios DX 80-200kV S/TEM. [2] Figure 1(a) lists key automation activities (using FEI's iFast Recipe Editor) that have been developed for EDS acquisition, quantification and metrology measurements. All measurements were obtained at $80 \mathrm{kV}$ on a probe-corrected Metrios DX.

Automated STEM/EDS metrology capabilities were evaluated using a commercially available 3D NAND memory device to compare critical dimensions to STEM images. Site navigation, feature alignment and tracking, layer alignment, focus, EDS mapping, on-line elemental quantification, and CD metrology were done entirely in automation using an iFast recipe. Active drift correction by beam shift and passive drift correction by frame integration were used to eliminate the effects of sample drift. Quantification of the 512 x 512 maps was done automatically at speeds more that 10x faster than manual routines in Esprit. Figures 1(b) and 1(c) are examples of a quantified EDS color map and individual grey scale elemental maps used for metrology measurements. Light elements ( $\mathrm{N}$ and $\mathrm{O}$ ), along with heavier elements, were detected with high contrast for robust metrology. The green arrows in Figure 1 (c) indicate regions of interest placed by an automated measurement recipe. The thickness of individual layers was obtained using an Edgefinder activity, which collapses a two dimensional spatial array of pixel intensities into a 1-dimensional plot of intensities as a function of distance. Figure 2 (a) shows STEM images acquired on the same ROI by automation. A robust recipe for measurement of NAND layers is very difficult with artifacts or features from various contrast forming mechanisms in all directions. There are no clear interfaces to resolve certain layers, such as N/Al/Ti layers. Figure 2 (b) highlights an example of automated EDS metrology measurements on individually extracted element maps, compared with STEM images from the same region. Unlike STEM/TEM images, EDS element 
maps are ideal candidates for metrology measurement as they have no other confounding contrast mechanisms except elemental distribution.

In this abstract we will report on the metrology precision and robustness of critical 3D NAND layers by EDS line scan and mapping compared to measurement of layers using other mechanisms of contrast formation (i.e. HAADF, bright-field, and dark-field).

\section{References:}

[1] Peter Schlossmacher et al, Microscopy and Analysis Nanotechnology Supplement (2010), p. s5.

[2] Ozan Ugurlu et al, SPIE Proceedings 8681 (2013), p. 868107.
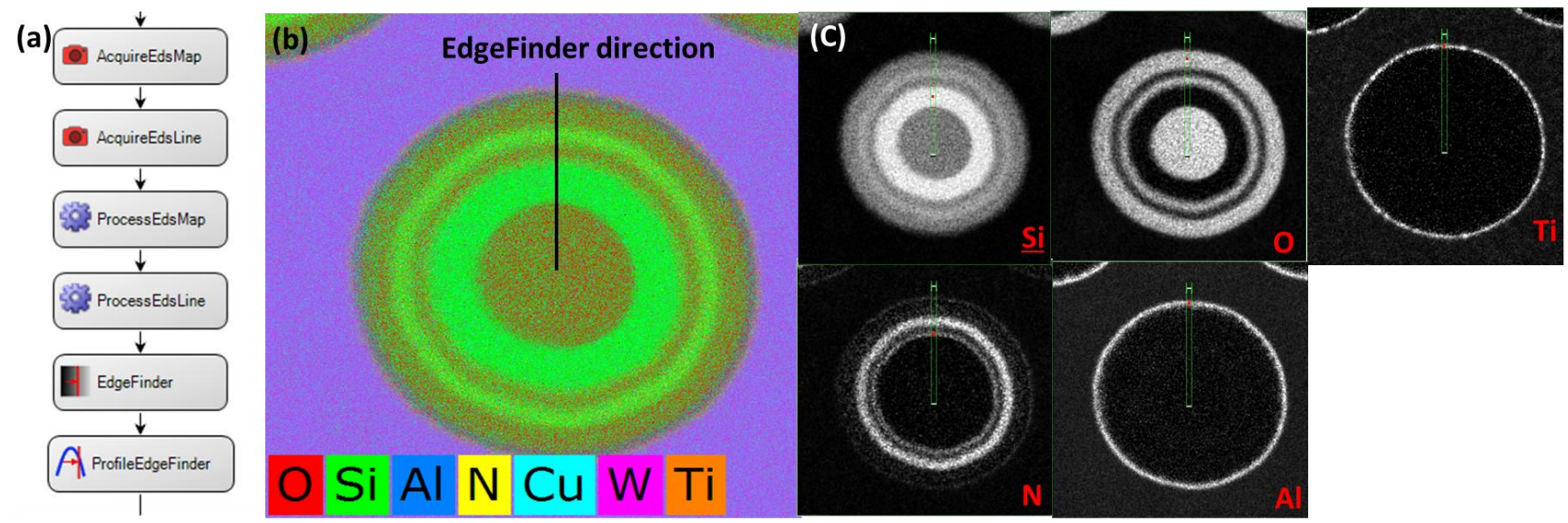

Figure 1. (a) Activities for automated EDS acquisition, quantification and metrology; (b) An example EDS color map by automatic acquisition and quantification; (c) Individual quantified elemental maps for EDS metrology. The green arrows in (c) are Edgefinders for metrology measurements. The field of view for both (b) and (c) images is $150 \mathrm{~nm}$.
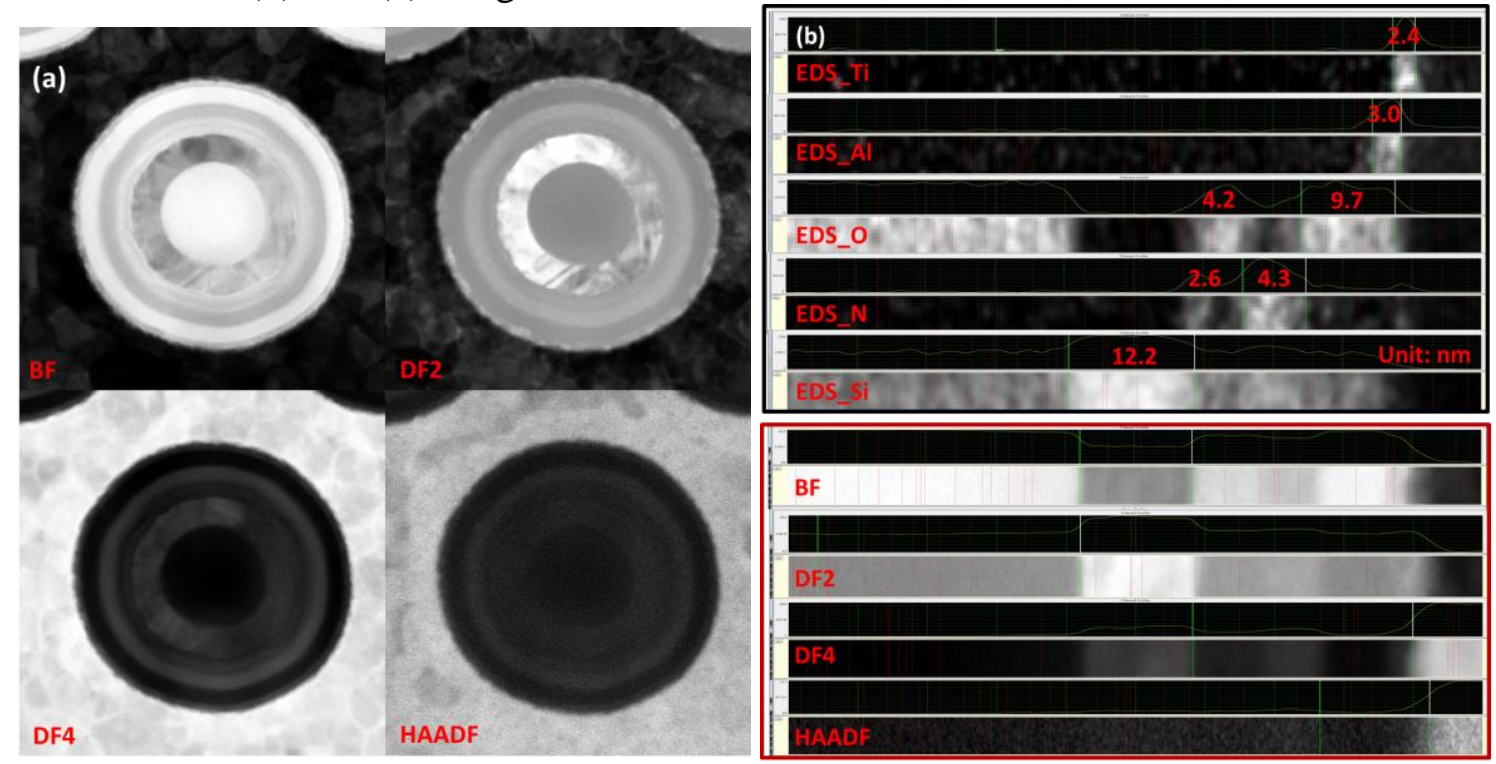

Figure 2. (a) STEM images acquired by automation from the same ROI for Figure 1(b) and 1(c); (b) A comparison of edge profiles from individual elemental maps (Ti, Al, O, N, and Si) and STEM images (BF, DF2, DF4, and HAADF). 